\title{
Capacity payment allocation in hydrothermal power systems with high shares of renewable energies
}

\author{
Carlos Benavides $^{1 *}$, Ricardo Álvarez ${ }^{1}$, Rigoberto Torres ${ }^{1}$, Rodrigo Moreno ${ }^{1}$, Marcelo Matus ${ }^{1}$, Francisco D. Muñoz ${ }^{2}$, José \\ M. González ${ }^{3}$, Guillermo Jiménez-Estévez ${ }^{1}$ and Rodrigo Palma-Behnke ${ }^{1}$ \\ ${ }^{1}$ Centro de Energía, Department of Electrical Engineering, University of Chile \\ ${ }^{2}$ Facultad de Ingeniería y Ciencias, Universidad Adolfo Ibánez \\ ${ }^{3}$ The University of Manchester, School of Engineering, Department of Mechanical, Aerospace and Civil Engineering (MACE)
}

\begin{abstract}
The increasing use of variable generation technologies (VGTs) in power systems, such as wind and photovoltaic generation, has introduced new challenges in the definition of capacity remuneration mechanisms for promoting system reliability. In this context, in this article we propose a novel framework for determining the capacity value of VGTs to contribute to the generation system adequacy, as well as a corresponding method for allocating capacity payments in hydrothermal power systems. We show that the capacity value of VGTs increases with the presence of water reservoirs, since VGTs help relieving hydropower plants from using scarce water resources during off-peak hours thus making more resources available to supply the demand during peak hours.
\end{abstract}

\section{Introduction}

Many countries around the world have committed to a decarbonisation of their electricity system. Under this global drive, variable generation technologies (VGT) such as wind power and photovoltaic generation appear as key pillars to achieve these energy targets and will undoubtedly play a key role in future power systems. Chile is not the exception. According to the country's Long-Term Energy Policy [1], the targets of the Chilean Government are that by the year 2035 at least $60 \%$ of the electrical generation must come from renewable sources, and $70 \%$ by the year 2050. The increasing use of VGTs has brought several countries to review their current capacity remuneration mechanisms, or design new ones, in order to account for the capacity value of VGTs. For instance, several works have estimated the capacity value of wind [2], [3], [4], photovoltaic (PV) [5], [6], and concentrating solar power (CSP) [7] resources. These work show that these renewables have capacity values that can range between $5 \%$ and $95 \%$ of the maximum generating capacity [8].

In Chile, the economic dispatch is performed in a centralized way by the Independent System Operator (Coordinador Eléctrico Nacional) with the aim of minimizing total system operating costs. Generating companies that participate in the electricity market are remunerated from energy sales, from providing available capacity and from the provision of ancillary services (frequency control, control voltage, service recovery, etc.). The valuation of the energy in the spot market is done on an hourly basis at the system marginal cost. A generation company that has a supply contract sells its energy at the contracted price. However, if at any point in time the generator is unable to fulfill its contracted commitment, it balances the deficit at the spot market, buying from companies that produce in excess. As for the capacity remuneration, each generator is recognized with a sufficiency capacity, which reflects the contribution of the generator to supply the peak load with a given probability and thus to contribute to the capacity sufficiency of the whole system. This concept excludes features that are remunerated as ancillary services. If a generating company has a contract with a client whose maximum demand is more than its recognized sufficiency capacity, then it must buy the difference in the capacity market from those generation companies that has an excess of capacity. The valuation of the sufficiency capacity within the capacity market is issued by the Chilean National Energy Commission (CNE), according to the investment cost of the most economical generation technology, which corresponds to a gas turbine. Therefore, if a power plants has a sufficiency capacity of $P_{d_{i}}$, it receives an annual income of $P_{d_{i}}(k W) \cdot C I\left(\frac{U S \$}{k W \cdot y e a r}\right)$, where $C I$ corresponds to the investment cost of the cutting-edge generatin technology.

The current methodology used in Chile for determining the sufficient capacity of each generator is shown in Fig. 1. At the beginning, each power plant is assigned with an Initial Capacity, which considers the availability of the primary energy source and is calculated using a conservative approach based on historic data. For example, the Initial Capacity of

*Corresponding author: cabenavi@centroenergia.cl 
hydropower plants is related to the availability of the water; whereas the Initial Capacity of thermal power plants is related to the availability of fossil fuels. For wind and solar power plants, the Initial Capacity is related to the wind and solar irradiation availability, respectively. In a second step, a preliminar sufficiency capacity is calculated using a probabilistic model. The preliminary sufficiency capacity $P S_{i}$ of each unit is calculated as the conditional expected value that a generator delivers a power $P_{i}$ given that the annual peak demand $D_{\max }$ is met. This can be mathematically expressed as: $P S_{i}=E\left(P_{i} \mid \sum_{i} P_{i}>D_{\text {max }}\right)$, where $P_{i}$ corresponds to the Initial Capacity. Finally, the definitive sufficiency capacity of each power plant $P_{d_{i}}$ is calculated based on preliminar sufficiency capacity of all generators and the maximum demand as follows: $P_{d_{i}}=$ $P S_{i} \cdot D_{\max } / \sum_{i} P S_{i}$.

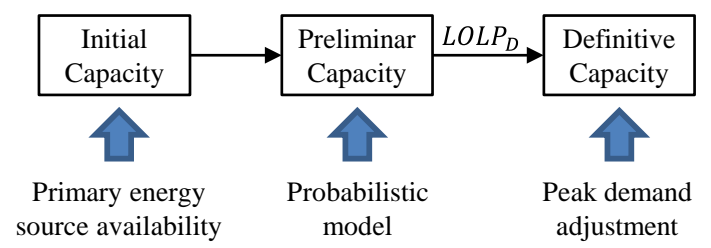

Fig. 1. Methodology used in Chile for estimating sufficiency capacity.

In Chile, hydroelectric power plants have a significant participation in the national electricity system. They represent $40 \%$ of the total generation, of which $18 \%$ have reservoirs. Therefore, a significant discussion was made on how to calculate the Initial Capacity of hydropower plants, given then high uncertainty surrounding the water availability. To illustrate this fact, Fig. 2 shows historical data of the equivalent energy flowing into the hydropower plants, ordered from the highest value to the lowest one. The two driest years occurred in the hydrologic years of 1968-1969 and 19981999, which are depicted in red. From this figure it can be seen that in the worst case the energy available form water resources is less than half than that of a wet year.

In order to follow a conservative approach and ensure system capacity sufficiency against worst case scenarios, the water availability considered for calculating the Initial Capacity of hydropower plants was determined as the average of the two worst hydrological years in terms of equivalent energy inflow. Later on, a similar approach was adopted for wind and solar power plants. In this case, the Initial Capacity was determined by multiplying the nominal power of each generator with the minimum value of: i) the lowest capacity factor of the past 5 years and ii) the average capacity factor observed in the 52 hours with the maximum load in the previous year. While i) is related to the annual energetic contribution of the wind or solar power plant, ii) is related to the contribution of the wind or solar power plant during peak hours. In this way, if a power plant has an annual capacity factor different from zero, but it did not produce energy during any of the 52 hours of peak demand, its initial capacity is zero.

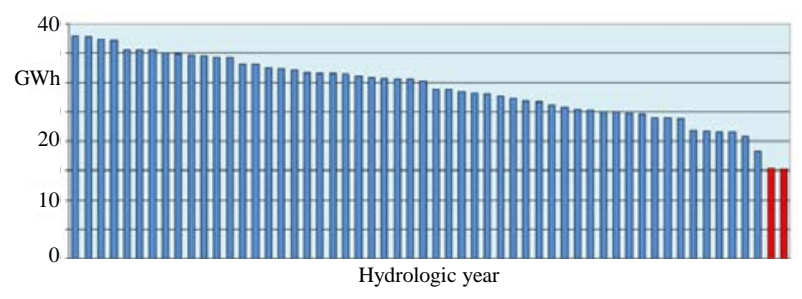

Fig. 2. Annual inflow energy into hydropower plants.

At an international level, there are several legislations that also use the generation feed-in of renewable energies in specific hours to determine the contribution to these type of generation technologies to the system sufficiency. In France, the contribution of wind and solar power plants to the system capacity sufficiency is calculated in ten hours time slots during the days with the highest probability of loss of load [9]. In California, the contribution to system sufficiency is calculated between 4 and $9 \mathrm{pm}$ from January to March and from November to December; and between 1 and 6 pm from April to October [10]. In the PJM System, the sufficient capacity for intermittent resources is based on calculating the average of the hourly output during the expected performance hours in the summer and winter. In summer (June to August) the expected performance hours are between $3 \mathrm{pm}$ and $8 \mathrm{pm}$, and in winter (January and February) between 6 am and 9 am and between $6 \mathrm{pm}$ and 9 [11]. An overview of modeling approaches regarding the development of electricity market design with a focus on capacity remuneration mechanisms or generation adequacy can be found in [12]. From the above it can be seen that the second component of the methodology used in Chile is coherent with other methods used in thermal power systems with significant shares of renewable energies. However, in this article we show that determining the capacity value of intermittent generating resources using as proxy the generation feed-in in a limited number of hours within a year may not be suitable in power systems with significant storage capacity, as is the case of Chile due to the presence of large water reservoirs.

The main contributions of this article are:

1.To propose a methodology for determining the contribution of VGT to the system capacity sufficiency in hydrothermal power systems

To show the results of applying the proposed methodology in a case study based on the Chilean National Electric System (SEN) and compare the results with the ones obtained using the current methodological approach followed in Chile

\section{Resource adequacy problem}

Generation adequacy is the ability of the generation in the power system to match the load at all times [13]. Two main indicators broadly used for adequacy assessments are the LOLP (loss of load probability) and the LOLE (loss of load expectation). The LOLP index is defined as the probability that power system outages leave the system with insufficient capacity to serve the 
load in a given period of time [8]. For a given time $t$, this probability index can be written as follows

$$
L O L P_{t}=\operatorname{Prob}\left\{G_{t}<L_{t}\right\}, \forall t=1, \ldots, T
$$

where $G_{t}$ and $L_{t}$ are the total available generation and demand at time $t$, respectively. The $L O L P$ is calculated by convolving the capacities and forced outages rates of the generation fleet together [2], and it usually includes stochastic models of the load and the availability of variable generating resources. The result of the LOLP index is expressed as the expected number of days in a year when a shortage might occur. A related reliability index is the $L O L E$, which is defined as the sum of $L O L P \mathrm{~s}$ over the planning horizon and gives the expected number of outage periods within that horizon. Mathematically, the $L O L E$ can be expressed as follows:

$$
L O L E=\sum_{t=1}^{T} \Delta t \cdot L O L P_{t}
$$

Since neither the $L O L P$ nor the $L O L E$ quantify the depth of the outage, a third index commonly used is the EENS (Expected Energy Not Supplied), which quantifies the amount of unsupplied energy during the period under study. The EENS can be computed as follows:

$$
\text { EENS }=\sum_{t=1}^{T} \Delta t \cdot L O L P_{t} \cdot P N S_{t}
$$

where $P N S_{t}$ corresponds to the unserved power in time $t$.

\subsection{Capacity payment estimation methods: marginal pricing}

As aforementioned, in Chile the energy sales in the spot market are valued at the system marginal cost, whereas the remuneration for capacity is based on a marginalist theory (peak-load pricing). The theoretical model is based on a deterministic optimization model that neglects the generation uncertainties. The optimal planning problem of the generation park can be modelled in a simplified way as follows:

$$
\min Z=\sum_{i=1}^{N G} a_{i} P_{i}+\sum_{i=1}^{N G}\left(b_{i} \sum_{j=1}^{N B} G_{i j} N_{j}\right)
$$

S.t

$$
\begin{gathered}
N_{j} \sum_{i=1}^{N G} G_{i j} \geq D_{j} N_{j}, j=1, \ldots, N B: \lambda_{j} \\
\sum_{i=1}^{N G} P_{i} \geq D_{\max }(1+M R T): \lambda_{0} \\
P_{i} N_{j}-G_{i j} \geq 0: \mu_{i} \\
P_{i}, G_{i j} \geq 0
\end{gathered}
$$

where $a_{i}$ represents the investment maintenance and operating costs (COMA) of unit $i$ in US\$/MW per year; $b_{i}$ represents the variable operating costs of unit $i$ in US $\$ / \mathrm{MWh} ; P_{i}$ is the installed capacity of unit $i$ in MW; $G_{i j}$ is the power generation of unit $i$ in block $j$ in MW; and $N_{j}$ Duration of the demand block $j$ in hours. The variables $\lambda_{0}, \lambda_{\mathrm{j}}$ and $\mu_{i}$ are the dual variables (shadow prices) associated with the restriction of the maximum demand, the energy restriction of block $\mathrm{j}$ and the generation restriction of plant $\mathrm{i}$, respectively. MRT represents the theoretical reserve margin.

The optimal expansion plan minimizes the annualized value of the investment, maintenance and operation (COMA) costs and the variable operating costs (4). Constraint (5) corresponds to the energy balance per block, (6) corresponds to the sufficiency restriction on the maximum demand; (7) and (8) are restrictions on the capacities and power generation.

Fig. 3 shows a load duration curve, discretized in blocks, along with the variables considered in the optimization problem (4)-(8).

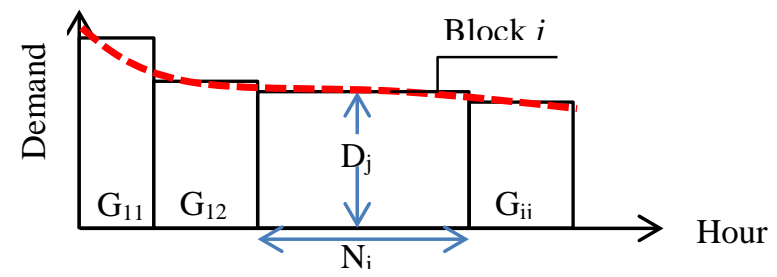

Fig. 3. Discretized load duration curve.

The objective function (4) can be written in its dual form as follows:

$$
\max z=\lambda_{0}^{*}(1+M T R) D_{\max }+\sum_{j=1}^{N B} \lambda_{j}^{*} D_{j} N_{j}
$$

In the optimum, the payment for power $\lambda_{0}^{*}(1+$ $M T R) D_{\max }$ and the payment for energy $\sum_{j=1}^{N B} \lambda_{0}^{*} D_{j} N_{j}$ equal the investment and operation cost of the system. The dual variable $\lambda_{0}^{*}$ corresponds to the marginal cost of increasing the system installed capacity, and the typical value used is the investment cost (in $U S \$ / \mathrm{kW}$ ) of a gas turbine, since it has the lowest investment cost. The variable $\lambda_{j}^{*}$ represents the marginal cost of the energy (in US\$/MWh) in each block. From this theory is derived that the payment for capacity is to be valued as the investment cost of the most economical technology.

\subsection{Capacity value estimation methods: Allocation schemes}

The capacity benefits of a given generating capacity addition can be expressed in terms of the increase in the system peak load carrying capability [14]. This factor is known as the ELCC index (Effective Load Carrying Capability). The ELCC essentially decomposes the contribution that an individual generator (or group of generators) makes to overall resource adequacy. A generator contributes to resource adequacy if it reduces 
the LOLP in some or all hours or days [2]. To compute the ELCC of a given generator, first the LOLE of the system is calculated without the capacity of the generator being evaluated $\left(L O L E^{B}\right)$, as follows:

$$
L O L E^{B}=\sum_{t=1}^{T} L O L P_{t}=\sum_{t=1}^{T} \operatorname{Prob}\left\{G_{t}<L_{t}\right\}
$$

When the generator being evaluated is considered within the LOLE calculation, it is expected a decrease in the $L O L E$, since the system has more installed capacity available. Therefore, in a second step the LOLE is again calculated, but this time considering the generator under evaluation and with an additional term for the demand $\bar{L}$, which is adjusted in order to obtain an equal value of the $L O L E$ as the one obtained without the contribution of the generator being evaluated, i.e. until $L O L E^{L}=L O L E^{B}$ :

$$
L O L E^{L}=\sum_{t=1}^{T} \operatorname{Prob}\left\{G_{t}+G_{t}^{g}<L_{t}+\bar{L}\right\}
$$

where $G_{t}^{g}$ corresponds to the available generation of generator $g$ at time $t$. In this way, the ELCC of the generator $g$ is defined as the increased demand $\bar{L}$ that can be achieved by incorporating the generation to the system, while maintaining the same level of $L O L E$.

Another alternative to measure the adequacy contribution of a power plant is by using the indices Equivalent Conventional Power $(E C P)$ or the Equivalent Conventional Capacity (ECC). The ECP or ECC of a generator $g$ is is defined as the capacity of a reference unit that can be replaced by the generator $g$ while maintaining the same LOLE. Normally, it is assumed that the reference unit has an expected failure rate greater than zero. To compute the $E C P$ or the $E C C$, the LOLE of the system including the generator $g$ is calculated as follows:

$$
L O L E^{g}=\sum_{t=1}^{T} \operatorname{Prob}\left\{G_{t}+G_{t}^{g}<L_{t}\right\}
$$

In a next step, the LOLE of the system including the reference generator and without the generator $g$ is calculated, as follows:

$$
L O L E^{B}=\sum_{t=1}^{T} \operatorname{Prob}\left\{G_{t}+B_{t}<L_{t}\right\}
$$

where $B_{t}$ is the available generation of the reference unit in $t$. The nominal capacity of the reference unit is adjusted iteratively until $L O L E^{g}=L O L E^{B}$. In this way, the ECP of the generator $\mathrm{g}$ is defined as the nominal capacity of the reference unit that achieves the previous equality.

\section{Proposed framework}

In this section we present a methodological approach for calculating the contribution of VGTs to system capacity sufficiency in hydrothermal power systems, and to determine the corresponding capacity payment allocation.

\subsection{Methodological approach and model}

Our proposed framework is based on the methodological approach used in Chile for determining the sufficient capacity of each generator presented in Section 1, and the marginal pricing method presented in Section 2.1. The proposed framework consists of solving the optimal generation expansion plan of the power system with and without considering VGTs. These results are used to determine the Initial Capacity of VGTs, which is defined as the difference between the installed capacities of the conventional generators obtained with and without considering the VGTS. Once the Initial Capacity of VGTs is obtained, we calculate the Preliminary Capacity and the Definitive Capacity according to the methodological approach used in Chile. Finally, the capacity payment is performed based on the Definitive Capacity of each generator. Fig. 4 shows an overview of the proposed framework.

The generation expansion planning model is similar to the one presented in (4)-(8), but adapted to its use in hydrothermal power systems. In this regard, the model includes the restrictions to represent the water balances in the reservoirs and, in order to correctly represent the use of the reservoirs, instead of using representative blocks for the demand, it considers all hours within a year. Notice that other types of storage can be easily included in the model as well.

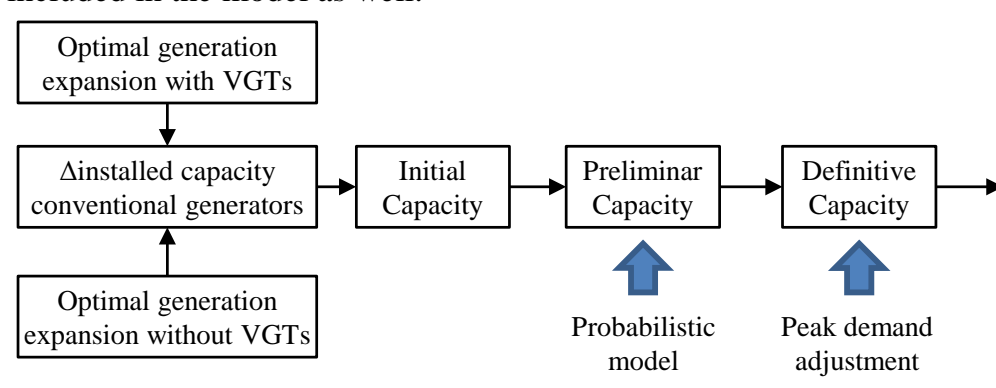

Fig. 4 Overview of the proposed framework

The optimal generation expansion problem can be written as follows:

$$
\min Z=\sum_{i=1}^{N G} a_{i} P_{i}+\sum_{i=1}^{N G} \sum_{t=1}^{8760} b_{i} G_{i j}
$$

s.t

$$
\begin{gathered}
\sum_{i=1}^{N G} G_{i t} \geq D_{t}, \forall t: \lambda_{1, t}^{*} \\
V_{\mathrm{it}}-V_{\mathrm{it}-1}+\mu_{i} G_{\mathrm{it}}+Q_{\mathrm{it}}=A_{\mathrm{it}}, \forall t, \forall i \in \Omega_{H}: \lambda_{2, y}^{*} \\
P_{i} f_{i t}-G_{i t} \geq 0, \forall t, \forall i \in \Omega_{V G T} \\
P_{i}, G_{i t} \geq 0, \forall t, \forall i \in \Omega_{G} \\
V_{\min } \leq V_{i t} \leq V_{\max }, \forall t, \forall i \in \Omega_{H}
\end{gathered}
$$


where $\Omega_{G}, \Omega_{H}$ and $\Omega_{V G T}$ are the sets containing all generators, the hydraulic power plants and the VGTs, respectively, and $N G$ is the total number of generators $\left(N G=\left|\Omega_{G}\right|\right)$. The objective function (14) consists of minimizing the system investment and operating costs. Constraint (15) represents the energy balance for each hour and the variables $\lambda_{1, t}^{*}$ are the corresponding Lagrange multiplier. The water balance of each hydropower with reservoir is presented in (16), where $\mu_{i}$ represents the convertion from electrical power to turbined water, $Q_{\mathrm{i} t}$ is a slack variable that represents the spilled water and $A_{\mathrm{i} t}$ is the inflow water. The variable $\lambda_{2, t}^{*}$ is the corresponding Lagrange multiplier. Constraint (17) limits the generation feed-in of VGTs (wind, solar and run-off-rivers) according to the hourly capacity factor $f_{i t}$, which can be computed based on historical profiles. Finally, constraints (18) and (19) impose limits to the power capacity and the generators, and in the water stored in the reservoirs, respectively.

The optimization problem (14)-(19) has the following assumptions: i) the capacity factors of VGTs are assumed known and fixed for each hour $t$; ii) the demand profile is deterministic; iii) no forced outage rates are considered; and iv) the theoretical reserve margin is zero.

Once the Initial Capacity of the VGTs are computed, the Preliminary Capacity and the Definite Capacity are calculated according to procedure described in Section 1. Finally, the allocation of the capacity payment among all generators is performed based on the Definitive Capacities obtained in the previous step. According to the marginalist theory, the investment and operating cost of all generating units should be recovered from energy sales at short term marginal price and from capacity payments. This monetary balance can be written as follows:

$$
\sum_{i=1}^{N G}\left(C I_{i}+C O P_{i}\right)=\sum_{i=1}^{N G} \sum_{t=1}^{T} S M C_{t} \cdot G_{i t}+I P^{S}
$$

where $C I_{i}$ and $C O P_{i}$ are the equivalent annual investment cost and the annual operating cost of power plant $i$, respectively; $S M C_{t}$ is the short-term marginal cost at hour $t$; $G_{i t}$ is the power generation of plant $i$ at hour $t$ and $I P^{S}$ is the total annual income for generation capacity. The total annual income for generation capacity to be allocated among the generators is:

$$
I P^{S}=\sum_{i=1}^{N G}\left(C I_{i}+C O P_{i}\right)-\sum_{i=1}^{N G} \sum_{t=1}^{T} C M G_{t} G_{i t}
$$

Assuming that the sufficiency capacity of each generator is valued at a fixed price $C P(U S \$ / M w)$, then the remuneration for sufficiency capacity for each generator is:

$$
I P_{i}^{S}=\sum_{i=1}^{N G} C P \cdot P_{i}^{S}
$$

where $P_{i}^{S}$ is the sufficiency capacity of generator $i$ (Definitive Capacity).

An important characteristic that any capacity payment method must fulfill is that it should not introduce any market distortions. In this regard, each power plant should be able to recover its investment and operating costs from energy sales and from the sufficiency capacity payment. This characteristic can be mathematically written as follows:

$$
I P_{i}^{S}=C P \cdot P_{i}^{s}=C I_{i}+C O P_{i}-\sum_{t=1}^{T} C M G_{t} G_{i t}
$$

The coherency of the proposed methodology with the marginalist theory can be analyzed by evaluating the fulfillment of (23) in the results obtained.

\section{Case Studies}

In this section we present the case studies carried out to i) validate the proposed methodology and analyze its results in an illustrative test case and ii) to show the sufficiency capacity value of VGTs in the Chilean Power System (SEN) obtained using the proposed methodology.

\subsection{Illustrative test case}

In this case, we implemented our proposed methodology in an illustrative test system that considers PV as VGT technology. A dry hydrological year was assume to reflect a worst case scenario regarding the system sufficiency. In addition, we considered two cases for the peak demand: a) the peak demand occurs between 12:00 and 14:00, i.e. when solar power is available; and b) the peak demand occurs at night, when no solar power is available.

Fig. 5 shows the dispatch in a representative weekly for the results obtained in case a), when the peak demand occurs during solar hours. The figure above shows the results obtained with $\mathrm{PV}$ and the figure below without $\mathrm{PV}$. The Equivalent Conventional Power (ECP) that is replaced by PV plants obtained with our proposed methodology was $1843 \mathrm{MW}$.

To compare the results obtained with other traditional approaches, we used the ELCC method to determine the increase in demand that can be achieved by forcing the entrance of the PV plants. In this case, the results were that the demand could be increase in $1842 \mathrm{MW}$ without $E E N S$, which is very similar to the PV capacity value obtained with our proposed methodology. Fig. 6 shows the increase in demand obtained with the ELCC method.

Finally, we calculated the capacity values of the generation park using the current normative in Chile. The results obtained for each case study are shown in Fig. 7. From this figure it can be seen that when the peak demand occurs during solar hours the results obtained with both methodologies are similar. However, when the peak demand occurs during the night, the capacity value of PV plants obtained using the current Chilean methodology is zero, while our methodology determines 


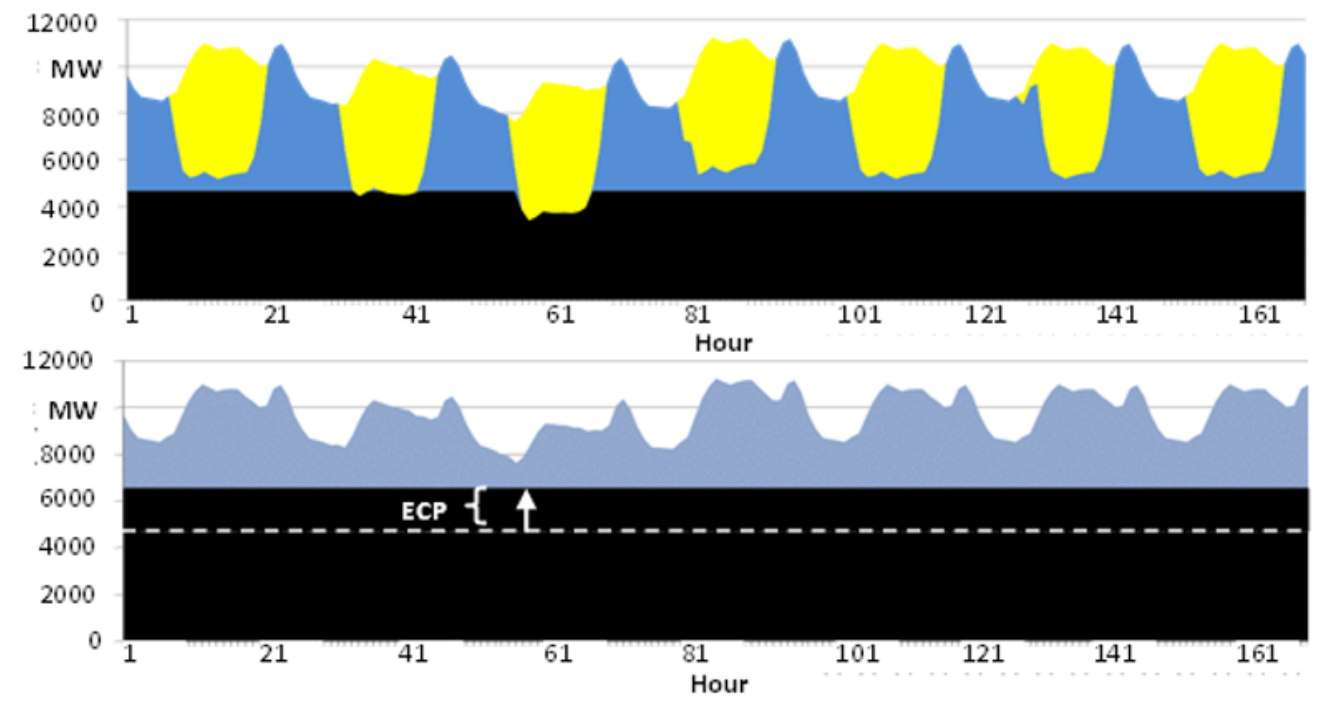

Fig. 5. Weekly dispatch for the results obtained in the cases 1 (thermal, hydro and PV) in the figure above, and 1a (thermal and hydro) in the figure below.

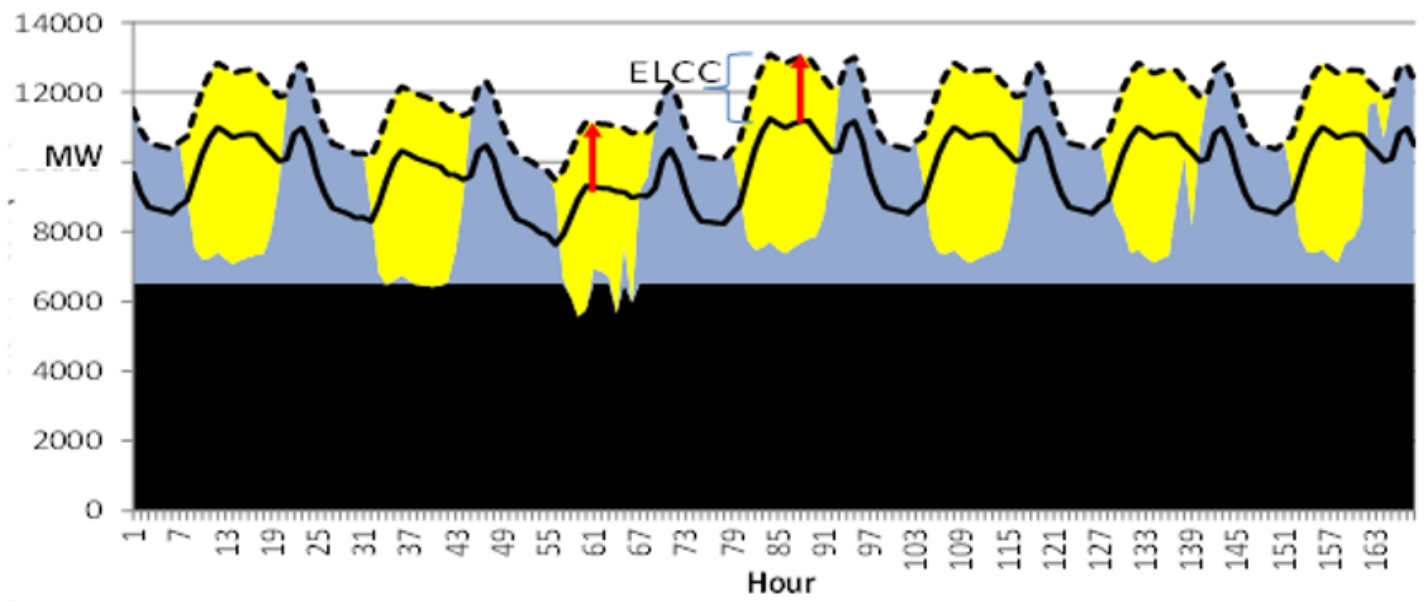

Fig. 6. Weekly dispatch for the results obtained in the cases 1 (thermal, hydro and PV) in the figure above, and 1a (thermal and hydro) in the figure below.

a capacity value of $1738 \mathrm{MW}$. The reason why our proposal values the contribution of PV to the system capacity sufficiency even though PV plants do not generate during peak demand hours, is because PV does contribute indirectly by relieving hydropower plants from using water resources for supplying the demand in off-peak hours. Notice that for this case study a dry hydrological year was assumed, and therefore the water resource is scarce. These results are coherent with the fact that PV plants do replace conventional generating capacity while maintaining the same level of system sufficiency. From these results it can be concluded that in hydrothermal power systems where the water resource undergo significant interannual variability and can be scarce, PV plants do contribute to the system sufficiency and should be therefore remunerated.

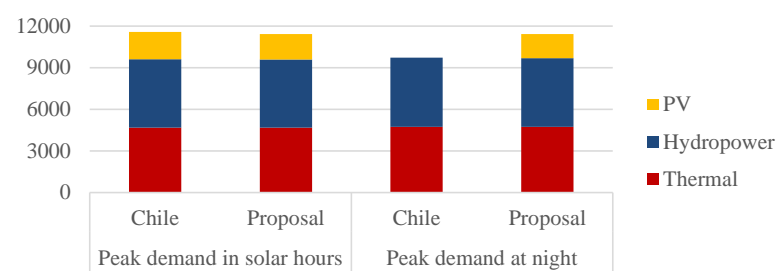

Fig. 7. Capacity values obtained with the current methodology used in Chile and with our proposal for different cases of peak demand occurrence.

\subsection{The Chilean case}

Finally, in this section we present the results obtained by applying the proposed methodology in the Main Chilean Power System (SEN) from April 2016 to March 2017. This period is chosen according to the hydrological year, which starts in April when the de-icing epoch begins. The installed capacity during that period was around 14 


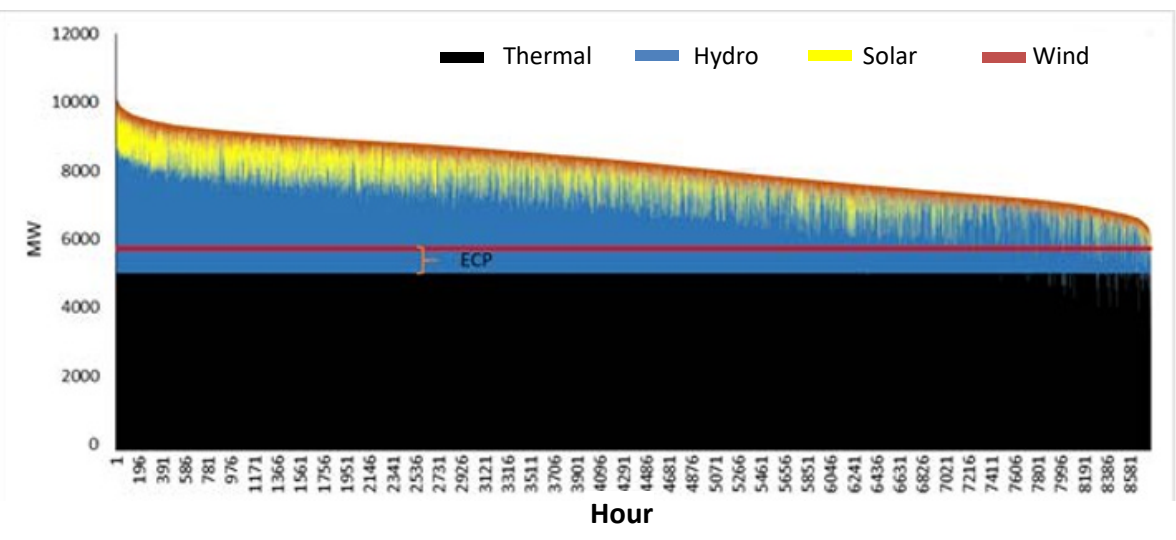

Fig. 8. Yearly duration curve during the period under study. The red horizontal line depicts the Initial Capacity for wind and PV obtained with our proposed methodology.

GW: $5 \mathrm{GW}$ of thermal power, $5.6 \mathrm{GW}$ of hydropower with reservoir, $1.8 \mathrm{GW}$ of solar power and $1.4 \mathrm{GW}$ of wind power. Fig. 8 shows the yearly duration curve during the period under study. The red horizontal line depicts the Initial Capacity for wind and PV obtained with our proposed methodology. The Initial Capacity for wind and PV was equal to $708 \mathrm{MW}$, which represents $21 \%$ of its installed capacity. This value is similar with the capacity factor obtained for these VGTs during the period under study, which was $22 \%$. By replacing the wind and PV feed-in profile for each plant with the ones with the minimum capacity factor obtained in the last 5 years, then the capacity value of wind and PV is reduced to $558 \mathrm{MW}$. This result shows that, even if worst case feed-in profiles in terms of yearly generation are assumed, our proposed methodology still values the contribution to the system sufficiency capacity of these VGTs.

Next we analyzed the impact of different penetration levels of VGT in the Initial Capacity Value. To this end, we fixed the demand and the hydrological scenario, and increased share of PV and wind energy. The results obtained are shown in Fig. 9. In this figure we also shows the results obtained for the Initial Capacity when implementing the capacity factor methodology. From this figure it can be seen that for low levels of VGT penetration (below 15\%) the results obtained with the proposed methodology are very similar to the ones obtained with the methodology based on the capacity factors. However, for higher penetration levels of VGTs, the sufficiency capacity value determined with our methodology starts being progressively less than the one obtained with the methodology based on the capacity factor. Finally, we analyzed the impact of the volumes of the reservoirs in the capacity value of VGTs. To this end, we reduced the storage capacity of each reservoir from its nominal value to zero. The results obtained in this case are shown in Fig. 10. From this figure it can be seen that, while the capacity value of wind is more or less independent from the storage capacity of the reservoirs, the capacity value of PV strongly depends on it, especially for low levels of storage capacity. The reason for this is because PV can only produce during day hours, and therefore the capacity value of PV strongly depends on the availability of storage capacity to shift energy from day hours to night hours and thus contribute to supply peak demand during night hours indirectly. Notice that up to a certain storage capacity (in this case, above 7200 million $\mathrm{m}^{3}$.

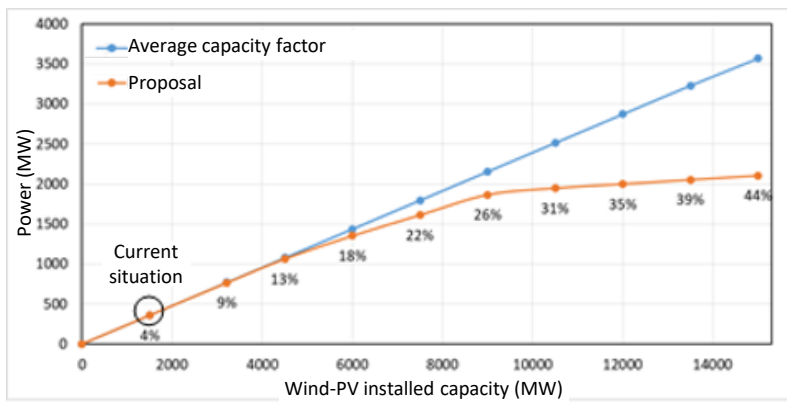

Fig. 9. Initial Capacity for PV and wind power plants in the SEN for different VGT penetration levels.

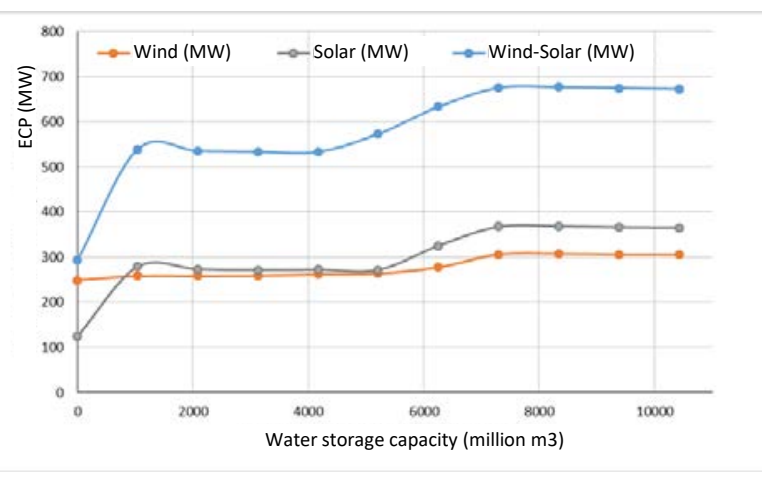

Fig. 10. Initial Capacity for PV and wind power plants in the SEN for different values of storage capacity in the reservoirs.

\section{Conclusions}

In this paper we propose a novel methodological approach for determining the contribution of VGTs to system capacity sufficiency in hydrothermal power systems. Furthermore, our proposed framework includes a method for capacity payment allocation that does not introduce market distortions. Our proposed framework is 
based on the methodological approach used in Chile and the marginal pricing method of peak-load-pricing. We showed that in the presence of reservoirs our proposal values the contribution of VGTs even when the generation does not occur in periods of peak hours. The main reason is that in these cases the VGTs do contribute indirectly to supply peak demand hours by relieving hydropower plants from using the limited water resources during off-peak hours, and use it instead during peak demand hours.

\section{References}

1. Gobierno de Chile, "Energía 2050. Política Energética de Chile,". Available: http://www.energia.gob.cl/sites/default/files/ energia_2050_-_politica_energetica_de_chile.pdf.

2. North American Electric Reliability Corporation (NERC), Methods to Model and Calculate Capacity Contributions of Variable Generation for Resource Adequacy Planning, Princeton, NJ, 2011.

3. A. Keane, M. R. Milligan, C. J. Dent, B. Hasche, C. D’Annunzio, K. Dragoon, H. Holttinen, N. Samaan, L. Soeder y M. O’Malley, "Capacity value of wind power," IEEE Trans. Power Syst., vol. 26, no 2, p. 564-572, 2011.

4. M. Milligan y K. Porter, "The Capacity Value of Wind in the United States: Methods and Implementation," The Electricity Journal, vol. 19, $\mathrm{n}^{\circ}$ 2, pp. 91-99, 2006.

5. National Renewable Energy Laboratory (NREL), "Update:Effective load-carrying capability of photovoltaics in the United States," CO, Tech. Rep. NREL/CP-620-40068, 2006.

6. C. Jilin, X. Qingshan, W. Xudong y J. Ling, "Estimation for the Capacity Value of PV and Wind Plants Considering Output Correlation," Energy Procedia, vol. 141, pp. 55-60, 2017.

7. S. Hossein, R. Sioshansi y P. Denholm, "Estimating the Capacity Value of Concentrating Solar Power Plants: A Case Study of the Southwestern United States," IEEE Trans. Power Syst., vol. 27, n 2, pp. 1116-1124, 2012.

8. S. Hossein, R. Sioshansi y P. Denholm, "Comparing Capacity Value Estimation Techniques for Photovoltaic Solar Power," IEEE Journal of Photovoltaics, vol. 3, n 1, pp. 407-415, 2013.

9. RTE, "French Capacity Market. Report accompanying the draft rules," 2014.

10. California ISO (CAISO), "Issue paper: Regional Resource Adequacy," 2015.

11. PJM, "PMJ Manual 18: PMJ Capacity Market," 2019.

12. A. Bublitz, D. Keles, F. Zimmermann, C. Fraunholz y W. Fichtner, "A survey on electricity market design: Insights from theory andreal-world implementations of capacity remuneration mechanisms," Energy Economics, vol. 80, p. 10591078, 2019.
13. M. Blanco, A. Spisto, N. Hrelja y G. Fulli, "Generation adequacy methodologies review," JRC Science for Policy Report, 2016.

14. R. Billinton, R. Karki, Y. Gao, D. Huang, P. Hu y W. Wangdee, "Adequacy Assessment Considerations in Wind Integrated Power Systems," IEEE Trans. Power Syst., vol. 27, no 4, pp. 22972305, 2012. 